\title{
Treating pneumonia in critical care in the United Kingdom following failure of initial antibiotic: a cost-utility analysis comparing meropenem with piperacillin/tazobactam
}

\author{
Steven J. Edwards • Sarah Wordsworth • \\ Mike J. Clarke
}

Received: 18 May 2010/Accepted: 3 January 2011/Published online: 18 January 2011

(C) Springer-Verlag 2011

\begin{abstract}
Background Treating patients admitted to critical care with severe pneumonia requires timely intervention with an effective antibiotic. This reduces the risk of dying of pneumonia and minimises complications associated with a prolonged stay in critical care.

Objective To compare the cost-effectiveness of meropenem $1 \mathrm{~g} / 8 \mathrm{~h}$ with piperacillin/tazobactam $4.5 \mathrm{~g} / 8 \mathrm{~h}$ for treating pneumonia in UK critical care.

Methods A Markov model was built to estimate lifetime costs and quality-adjusted life years (QALYs) of using meropenem versus piperacillin/tazobactam to treat severe pneumonia. Estimates of effectiveness, utility weights and costs were obtained from published sources. Probabilistic sensitivity analysis was conducted to address uncertainty in the model results.

Results Cost of treating a patient with severe pneumonia was estimated as $£ 19,026$ with meropenem and $£ 19,978$ with piperacillin/tazobactam, respectively. QALYs gained were 4.768 with meropenem and 4.654 with piperacillin/ tazobactam. Probabilistic sensitivity analysis showed meropenem to be consistently less costly and more effective than piperacillin/tazobactam.
\end{abstract}

S. J. Edwards $(\bowtie)$

Kellogg College, University of Oxford, 62 Banbury Road, Oxford OX2 6PN, UK

e-mail: steven.edwards@kellogg.ox.ac.uk

S. Wordsworth

Health Economics Research Centre, Department of Public

Health, University of Oxford, Oxford, UK

M. J. Clarke

UK Cochrane Centre, National Institute for Health Research,

Oxford, UK
Conclusion The additional efficacy of meropenem translates into more patients surviving critical care and leaving this high-cost service more quickly than if they had been treated with piperacillin/tazobactam. As meropenem is more effective and less expensive than piperacillin/tazobactam at treating patients with severe pneumonia, it is the dominant treatment option.

Keywords Meropenem - Piperacillin · Infection · Critical care $\cdot$ Cost-effectiveness analysis .

Economic evaluation

JEL Classification L65

\section{Introduction}

Infection in hospital is defined as community-acquired (i.e. infection contracted outside the health care setting or present on admission) or nosocomial (i.e. first appearing $48 \mathrm{~h}$ or more after hospital admission). If a patient's infection is serious enough to require admission to critical care, this tends to be because the infection itself is lifethreatening, or because the patients existing condition makes any infection potentially life-threatening. A recent assessment of critical care admissions in England, Wales and Northern Ireland identified the proportion of patients admitted to critical care units with community-acquired pneumonia as a relatively stable $6.4 \%$ (around 8,000 admissions per annum) [1]. Community-acquired pneumonia is the leading cause of death from infection internationally and the sixth leading cause of death overall [2].

Current guidelines (e.g. the American Thoracic Society, British Thoracic Society, etc.) for the antibiotic treatment of community or hospital-acquired pneumonia recommend 
beta-lactams for initial empirical therapy [3-6], in particular beta-lactams with a spectrum of activity against both grampositive (such as Staphylococcus aureus, streptococci and pneumococci) and gram-negative (such as Pseudomonas aeruginosa and Enterobacter spp.) pathogens. These beta-lactams have been identified as the 4th generation cephalosporins (cefepime and cefpirome), the carbapenems (imipenem/cilastatin and meropenem) and the antipseudomonal penicillins (piperacillin/tazobactam and ticarcillin/ clavulanate) [7].

In the United Kingdom, the antibiotics typically used in critical care are a carbapenem (imipenem/cilastatin or meropenem) and piperacillin/tazobactam. Most critical care units select imipenem/cilastatin or meropenem as their carbapenem of choice to list on their formulary, rather than having both antibiotics available. The results from a previous study showed that meropenem $1 \mathrm{~g} / 8 \mathrm{~h}$ is a cost-effective alternative to imipenem/cilastatin $1 \mathrm{~g} / 8 \mathrm{~h}$ [8], and so meropenem could be considered the preferred carbapenem unless other factors affect this decision (such as local pathogen resistance).

However, the appropriate prescribing of meropenem compared to piperacillin/tazobactam has not been the subject of economic evaluation. This may be due to the absence of randomised trials directly comparing the two antibiotics in the treatment of severe infection [9]. We have previously performed an adjusted indirect comparison using a mixed treatment comparison [10], which demonstrated substantial clinical benefits with meropenem over piperacillin/tazobactam in the treatment of patients hospitalised with infection [11].

In addition to the clinical benefits meropenem may have over piperacillin/tazobactam, it must be evaluated in the light of any additional increase in resource utilisation. Compared to other departments within a hospital, it has been identified that drug therapies used in critical care can have a substantial increase in costs [12]. We decided to conduct a study aimed at estimating the cost-effectiveness of meropenem compared to piperacillin/tazobactam in the treatment of pneumonia in UK critical care. These results could be used to determine which treatment strategy offers the best value for money from National Health Service (NHS) resources.

\section{Methods}

The perspective for the evaluation was the UK NHS, since the costs associated with the use of meropenem or piperacillin/tazobactam and subsequent treatment lie predominantly within a hospital care setting. All costs are for the price year 2008. A Markov cohort model was used to estimate the average patient experience and to assess the parameter uncertainty. The model was constructed using TreeAge Pro $2005^{\mathrm{TM}}$.
Figure 1 shows the underlying treatment algorithm informed by a survey of five consultant microbiologists and four intensive care consultants based at Bedford Hospital; John Radcliffe Hospital in Oxford; Leeds General Infirmary; Gloucestershire Royal Hospital; Northampton General Hospital; and Royal Berkshire Hospital. These hospitals are geographically spread throughout England and are a mixture of teaching hospitals and district general hospitals. The survey was conducted by one of the authors by individual interviews with the clinicians. A consensus report was created based on the narrative descriptions of care provided. Three iterations of the report were circulated to the clinicians involved before a final version was agreed by all respondents.

At the start of the model (Fig. 1), patients with severe pneumonia are assumed to be in an intensive care unit (ICU) having failed on first-line antibiotics (either before or after admission to ICU). The severity of illness is simulated in the model by those requiring mechanical ventilation using a ventilator (more severe) and those not requiring mechanical ventilation (less severe). Regardless of severity, all patients receive intravenous piperacillin/ tazobactam or meropenem for a minimum of 5 days and a maximum of 14 days.

During antibiotic treatment, patients can experience severe adverse events and are at risk of contracting other infections while in critical care. However, the model only considers two of these: Clostridium difficile-associated diarrhoea and the risk of acquiring ventilator-associated pneumonia (VAP). These are associated with a substantial impact on treatment choice and/or length of stay, as well as risk of death. Patients may die at any time in critical care.

Patients who have $C$. difficile-associated diarrhoea have their antibiotic changed to the alternative treatment (i.e. patients initially receiving piperacillin/tazobactam switch to meropenem and vice versa), and metronidazole $250 \mathrm{mg}$ every $6 \mathrm{~h}$ for 10 days is added to their treatment. After 10 days of treatment with metronidazole, patients are assumed to be cured of $C$. difficile and may leave ICU once they have a clinical response with meropenem or piperacillin/tazobactam.

Patients who acquire VAP are treated with a combination of ceftazidime ( $3 \mathrm{~g}$ every $12 \mathrm{~h}$ ) and gentamicin $(5 \mathrm{mg} / \mathrm{kg}$ every $24 \mathrm{~h}$ ) for 8 days. After 8 days of treatment, patients are assumed to be cured of VAP and their underlying pneumonia but require additional time in ICU to recover.

After 5 days of treatment with piperacillin/tazobactam or meropenem, patients may have a clinical response and be moved to a high dependency unit (HDU), respiratory/ general ward, or discharged from hospital. To simulate 5 days of treatment as the number of days required for clinical response, and the most common duration of treatment, the transition probability was calculated per day for 


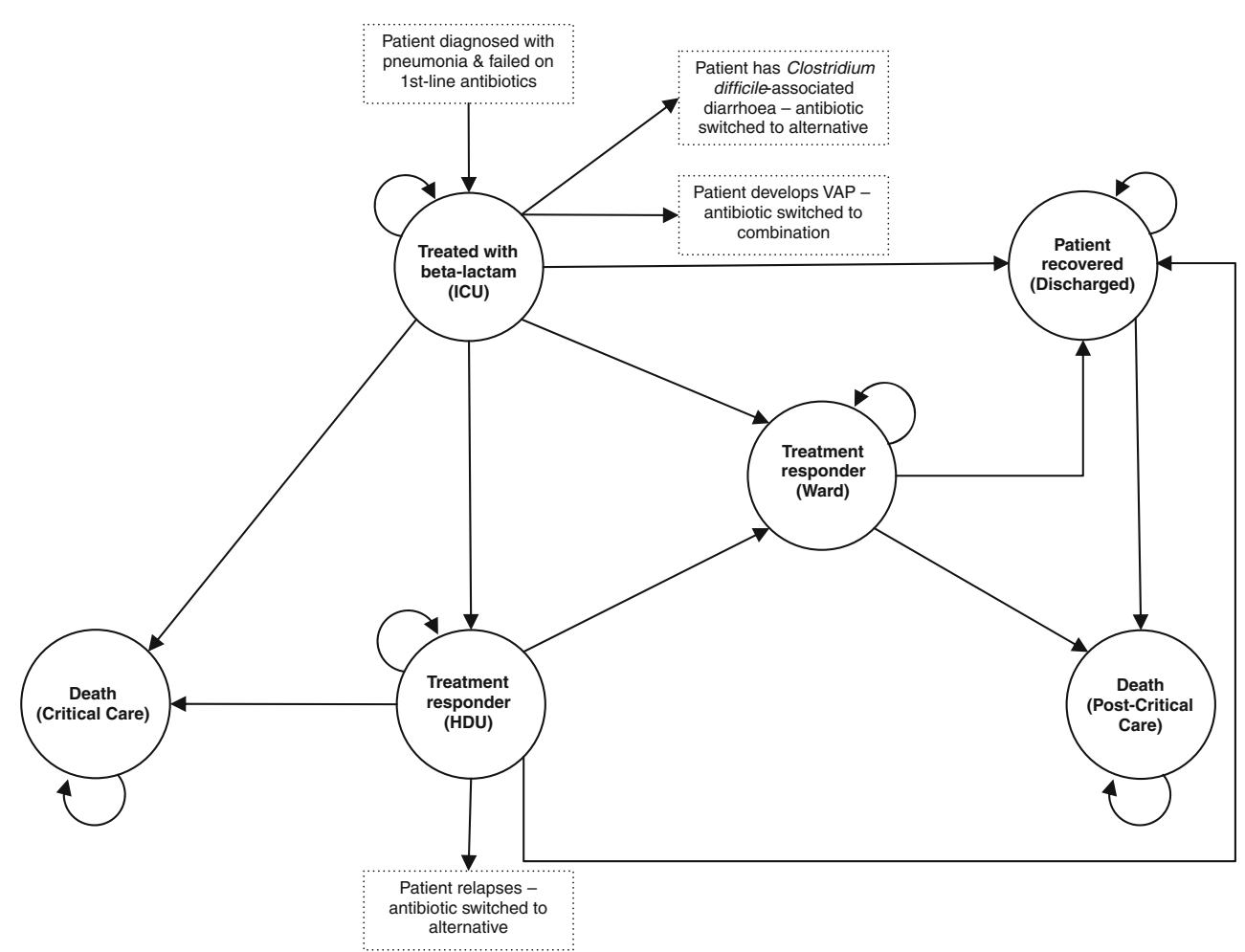

Fig. 1 Treatment pathway for patients with pneumonia in critical care. The circles reflect locations of care, such as ICU, HDU, ward, etc., with patients remaining in each location unless they respond to treatment, have an adverse event (including relapse) or die

the maximum number of days of treatment (14 days) and then the daily value was multiplied by 5 for those initial 5 days of treatment.

Patients moved to HDU are assessed to see whether they still require mechanical ventilation, fluid management or both. Once considered stable, they are either moved to a respiratory/general ward or discharged. Some patients may have their pneumonia recur and relapse back into ICU.

Patients who relapse back into ICU have their antibiotic changed to the alternative treatment (i.e. patients who initially received piperacillin/tazobactam will receive meropenem and vice versa) and begin treatment again. Patients who relapse back into ICU have an increased risk of dying in critical care.

Once a patient arrives on a respiratory/general ward, they remain there until they recover sufficiently for discharge home. As a patient has left critical care, they no longer have the high risk of dying associated with being in ICU or HDU. However, they do have a higher annual rate of mortality than normal (age- and sex-adjusted) due to having been in critical care.

Patients discharged from hospital are assumed to have a single follow-up outpatient appointment. They can either live or die in accordance with the appropriate age- and sexadjusted annual rate of mortality, which is increased in the initial years after discharge, because they have been ill enough to be in critical care.
Transition probabilities

The parameters used within the Markov model are separated into five different categories:

- Common-applicable to meropenem or piperacillin/ tazobactam;

- Efficacy-specific to meropenem or piperacillin/ tazobactam;

- Utility—preference for a given health state independent of treatment;

- Drug costs-costs of medication and administration;

- Service costs-costs associated with level of care.

The parameter estimates used in the base-case model are presented in Table 1. Transition probabilities were calculated from the original data using the method described by Sun and Faunce [33].

Common parameter estimates (Table 1a) were obtained from either a systematic review of that parameter, evidencebased guidelines or an analysis of Hospital Episode Statistics (HES) [20]. Efficacy parameters (Table 1b) were based on a previously completed mixed treatment comparison [11] with a baseline for piperacillin/tazobactam obtained from a relevant randomised trial [25]. Pertinent details of the patient population in that trial are presented in Table 2.

Utility parameters (Table 1c) were estimated from the best available data. However, there has been very little 
Table 1 Parameter estimates used in the health economic model evaluating the cost-effectiveness of meropenem compared with piperacillin/ tazobactam in the treatment of patients with severe pneumonia requiring admission to a critical care unit (CCU)

\begin{tabular}{|c|c|c|}
\hline Parameter & Original data & Transition probability \\
\hline \multicolumn{3}{|l|}{ a Common parameters } \\
\hline Patient ventilated [13] & $53 \%(\mathrm{SD} 3 \%)$ & 0.530000 \\
\hline Diarrhoea being Clostridium difficile related [14] & $7 \%(\mathrm{SD} 1 \%)$ & $\begin{array}{l}\text { Not applicable-applied directly } \\
\text { to probability of diarrhoea }\end{array}$ \\
\hline Patient cured of $C$. difficile [15] & All assumed cured at 10 days & $\begin{array}{l}\text { Tunnel state with } 100 \% \text { at } 10 \text { th cycle } \\
\text { after entering health state }\end{array}$ \\
\hline Patient cured of VAP [16] & All assumed cured at 8 days & $\begin{array}{l}\text { Tunnel state with } 100 \% \text { at } 8 \text { th cycle } \\
\text { after entering health state }\end{array}$ \\
\hline Dying in CCU if ventilated [13] & $44 \%(\mathrm{SD} 5 \%)$ & 0.069913 \\
\hline Dying in CCU if not ventilated [13] & $1 \%(\mathrm{SD} 1 \%)$ & 0.001256 \\
\hline Dying in CCU with VAP [17] & $44 \%(\mathrm{SD} 5 \%)$ & 0.069913 \\
\hline $\begin{array}{l}\text { Adjustment for death from VAP } \\
\text { based on age of patient [18] }\end{array}$ & $\begin{array}{l}>59 \text { years OR5.36 (95\% CI 1.64-17.6) } \\
>79 \text { years OR11.8 (95\% CI 2.99-46.5) }\end{array}$ & $\begin{array}{l}\text { OR converted to RR applied directly } \\
\text { to probability of dying in critical } \\
\text { care with VAP }\end{array}$ \\
\hline \multirow[t]{4}{*}{ Developing VAP [19] } & Within $24 \mathrm{~h} ; 7 \%$ (SD2\%) & 0.020300 \\
\hline & $24-48 \mathrm{~h} ; 6 \%(\mathrm{SD} 2 \%)$ & 0.017400 \\
\hline & $2-6$ days; $1 \%(\mathrm{SD} 1 \%)$ & 0.002509 \\
\hline & $>6$ days; $3 \%(\mathrm{SD} 1 \%)$ & 0.007586 \\
\hline $\begin{array}{l}\text { Adjustment in risk of VAP infection } \\
\text { based on prior antibiotic use [19] }\end{array}$ & $\begin{array}{l}\leq 48 \text { h: OR } 0.29,95 \% \text { CI } 0.12-0.69 \\
>48 \text { h: OR } 1.47, p<0.05\end{array}$ & $\begin{array}{l}\text { OR converted to RR applied directly } \\
\text { to probability of developing VAP }\end{array}$ \\
\hline Recovering from VAP [16] & 22 days (SD20.0) & 0.113906 \\
\hline Bed days in HDU [20] & 5.0 days (SD5.7) & 0.206299 \\
\hline Leaving ICU for HDU [20] & $31.1 \%(\mathrm{SD} 23.1 \%)$ & 0.311000 \\
\hline Leaving ICU for ward [20] & $67.7 \%(\mathrm{SD} 22.7 \%)$ & 0.677000 \\
\hline Leaving ICU for discharged [20] & $1.2 \%(\mathrm{SD} 2.4 \%)$ & 0.012000 \\
\hline Leaving HDU for ward [20] & $96.9 \%(\mathrm{SD} 3.8 \%)$ & 0.969000 \\
\hline Leaving HDU for discharged [20] & $3.1 \%(\mathrm{SD} 3.8 \%)$ & 0.031000 \\
\hline Discharge from hospital [20] & 24.8 days (SD23.2) & 0.041139 \\
\hline \multirow{6}{*}{$\begin{array}{l}\text { Normalised age-related death following } \\
\text { discharge from CCU [21] }\end{array}$} & $65-691.6 \%$ & 0.000038 \\
\hline & $70-742.6 \%$ & 0.000066 \\
\hline & $75-794.4 \%$ & 0.000112 \\
\hline & $80-847.5 \%$ & 0.000192 \\
\hline & $85-8912 \%$ & 0.000315 \\
\hline & $9022.4 \%$ & 0.000605 \\
\hline \multirow{9}{*}{$\begin{array}{l}\text { Adjustment for death following } \\
\text { discharge from CCU based } \\
\text { on increased risk following } \\
\text { CCU admission [22] }\end{array}$} & $\begin{array}{l}\text { Post-CCU increased mortality per year (RR): } \\
1 \text { st } 15.26,95 \% \text { CI } 11.56-20.17\end{array}$ & \multirow[t]{9}{*}{$\begin{array}{l}\text { Applied directly to probability of dying } \\
\text { in critical care }\end{array}$} \\
\hline & 2nd $2.55,95 \%$ CI $1.69-3.86$ & \\
\hline & 3rd $2.05,95 \%$ CI $1.33-3.17$ & \\
\hline & 4th $1.68,95 \%$ CI $1.07-2.66$ & \\
\hline & 5th $1.51,95 \%$ CI $0.95-2.40$ & \\
\hline & 6th $1.22,95 \%$ CI $0.73-2.03$ & \\
\hline & 7 th $0.77,95 \%$ CI $0.41-1.46$ & \\
\hline & 8th $0.62,95 \%$ CI $0.30-1.29$ & \\
\hline & 9th $1.05,95 \%$ CI $0.51-2.18$ & \\
\hline Relapsing back into ICU [23] & $5.6 \%(\mathrm{SD} 0.1 \%)$ & 0.019026 \\
\hline $\begin{array}{l}\text { Adjustment for death following } \\
\text { readmission to ICU [24] }\end{array}$ & OR 11.0 (95\% CI: 8.1-15.1) & $\begin{array}{l}\text { OR converted to RR applied to } \\
\text { probability of dying in critical care }\end{array}$ \\
\hline
\end{tabular}


Table 1 continued

\begin{tabular}{|c|c|c|}
\hline Parameter & Original data & Transition probability \\
\hline \multicolumn{3}{|l|}{ b Efficacy parameters } \\
\hline Diarrhoea on meropenem [11] & OR 2.60 (95\% CrI 0.9793-5.723) & $\begin{array}{l}\text { OR converted to RR applied } \\
\text { to piperacillin/tazobactam baseline }\end{array}$ \\
\hline Clinical response with meropenem [11] & OR 0.5059 (95\% CrI 0.3492-0.7089) & $\begin{array}{l}\text { OR converted to RR applied } \\
\text { to piperacillin/tazobactam baseline }\end{array}$ \\
\hline Diarrhoea on piperacillin/tazobactam [25] & $10.0 \%$ & 0.007498 \\
\hline Clinical response with piperacillin/tazobactam [25] & $69.1 \%$ & 0.080445 \\
\hline
\end{tabular}

Utility Parameters $\quad$ Original data uaily utilies

\begin{tabular}{|c|c|c|c|c|c|c|}
\hline \multicolumn{7}{|c|}{ c Utility parameters } \\
\hline \multicolumn{4}{|c|}{$\begin{array}{l}\text { Utility in critical care centres } \\
\text { (ICU and HDU) [26] }\end{array}$} & \multicolumn{2}{|c|}{$\begin{array}{l}\text { Unconscious }-0.402 \text { (Worst [33333] } \\
-0.594 \text { to Best }[33311] 0.028)\end{array}$} & -0.001101 \\
\hline \multicolumn{4}{|c|}{ QALY recovering on ward [27] } & \multicolumn{2}{|c|}{$0.726317^{\mathrm{a}}$} & 0.001990 \\
\hline \multicolumn{4}{|c|}{ QALY 65-74 [26] } & \multicolumn{2}{|l|}{$0.78(\mathrm{SD} 0.28)^{\mathrm{b}}$} & 0.002137 \\
\hline \multicolumn{4}{|c|}{ QALY 75+ [26] } & \multicolumn{2}{|l|}{$0.71(\mathrm{SD} 0.27)^{\mathrm{b}}$} & 0.001945 \\
\hline \multirow[t]{2}{*}{ Parameter } & \multicolumn{4}{|c|}{ Administration [29] } & \multicolumn{2}{|l|}{ Drug cost $[30]$} \\
\hline & Delivery & Daily cost ${ }^{\mathrm{c}}$ & Original data $^{\mathrm{d}}$ & Dosing regimen & Daily cost & Original data \\
\hline \multicolumn{7}{|c|}{ d Drug cost and administration parameters } \\
\hline Meropenem & Intravenous & $£ 10.37$ & $€ 4.35+16.52 \mathrm{~min}$ & $1 \mathrm{~g} / 8 \mathrm{~h}$ & $3 \mathrm{~g}=£ 51.57$ & $1 \mathrm{~g}$ vial $£ 17.19$ \\
\hline Pip/Tazo & Intravenous & $£ 10.37$ & $€ 4.35+16.52 \mathrm{~min}$ & $4.5 \mathrm{mg} / 8 \mathrm{~h}$ & $13.5 \mathrm{mg}=£ 47.37$ & $4.5 \mathrm{mg}$ vial $£ 15.79$ \\
\hline Metronidazole & Intravenous & $£ 6.11$ & $€ 4.35+5.40 \mathrm{~min}$ & $500 \mathrm{mg} / 8 \mathrm{~h}^{\mathrm{e}}$ & $1,500 \mathrm{mg}=£ 3.06$ & $\begin{array}{l}100 \mathrm{~mL} \text { amp } £ 8.17 \\
(200 \mathrm{mg} / 5 \mathrm{~mL})\end{array}$ \\
\hline $\begin{array}{l}\text { Ceftazidime and } \\
\text { Gentamicin }\end{array}$ & Intravenous & $£ 8.86$ & $\begin{array}{l}€ 4.35+5.40 \text { and } \\
€ 1.45+3.65 \text { min }\end{array}$ & $\begin{array}{l}\text { Ceftazidime } 2 \mathrm{~g} / 8 \mathrm{~h} \\
\text { Gentamicin } \\
5 \mathrm{mg} / \mathrm{kg} / \text { day }\end{array}$ & $\begin{array}{l}\text { Ceftazidime } 6 \mathrm{~g}=£ 53.70 \\
\text { Gentamicin } 350 \mathrm{mg}=£ 6.48 \\
\text { Ceftazidime }+ \text { Gentamicin }=£ 60.18\end{array}$ & $\begin{array}{l}\text { Ceftazidime: } 2 \text { g vial } \\
£ 17.90 \text { Gentamicin: } \\
2 \mathrm{ml} \text { vial }(40 \mathrm{mg} / \mathrm{ml}) \\
£ 1.48\end{array}$ \\
\hline
\end{tabular}

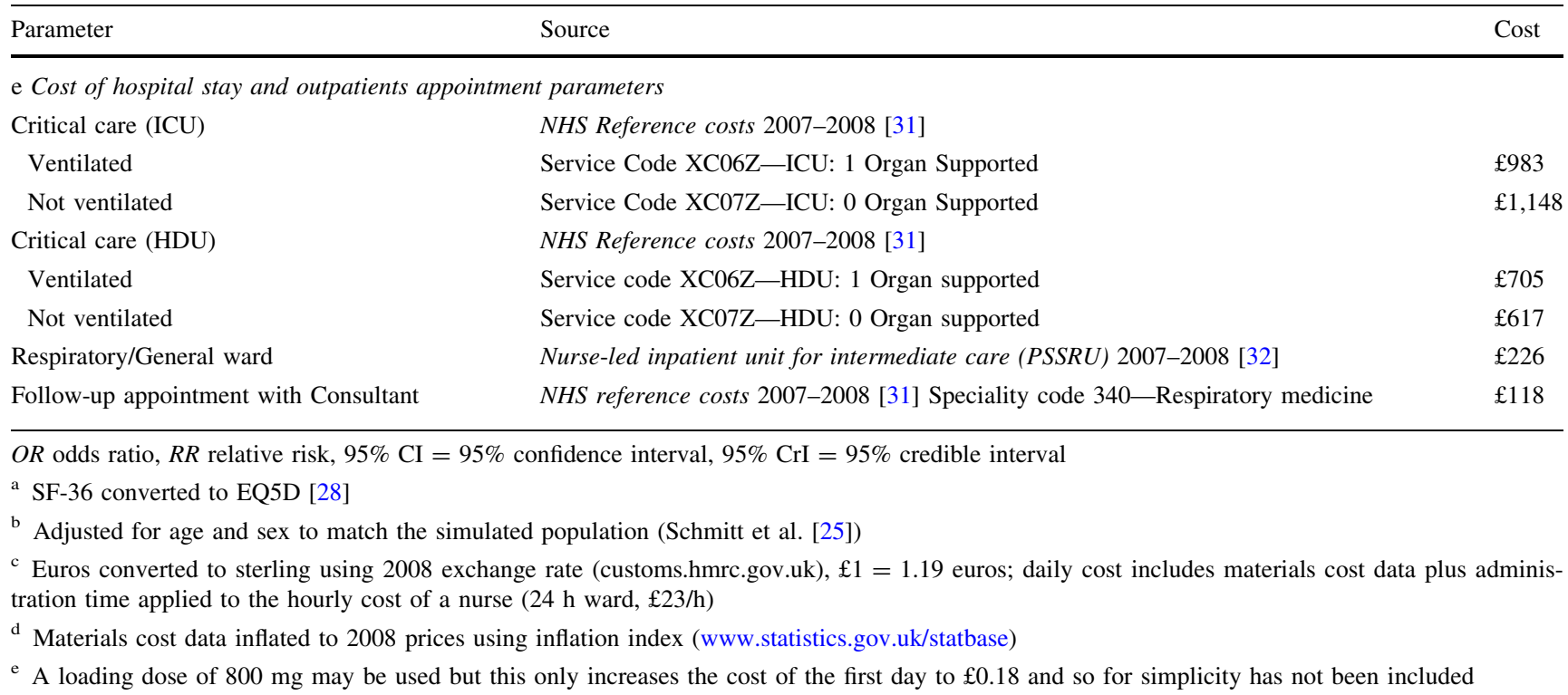

health-related quality of life (HRQL) research conducted in critical care [34]. In the base-case analysis, it was assumed that patients in critical care (ICU and HDU) had the utility score from the EuroQoL (EQ-5D) social tariff for "unconscious" [26]. Utilities applied for patients recovering on the ward after discharge from critical care were obtained from a published survey [27]. Once patients were discharged from hospital, it was assumed that they accrued 
Table 2 Baseline characteristics of patients simulated in the Markov model based on patients randomised to piperacillin/tazobactam in Schmitt et al. [25]

\begin{tabular}{ll}
\hline Characteristic & Population \\
\hline Age (SD) & $68.4(13.7)$ years \\
Male/Female & $70 \% / 30 \%$ \\
APACHE II score (SD) & $13.5(4.2)$ \\
Abnormal X-ray & $98.2 \%$ \\
Body temperature & \\
$\geq 38^{\circ} \mathrm{C}$ & $67.3 \%$ \\
$36.1-37.9^{\circ} \mathrm{C}$ & $31.8 \%$ \\
Leukocytosis & $69.1 \%$ \\
C-reactive protein & \\
$\leq 15 \mathrm{mg} / \mathrm{l}$ & $8.2 \%$ \\
$>15 \mathrm{mg} / 1$ & $79.1 \%$ \\
\hline
\end{tabular}

their normal age- and sex-adjusted HRQL as provided by the EQ-5D [26].

The cost of drug administration (Table 1d) was taken from Van Zanten and colleagues [29] because it provided data on three of the four interventions with only a single assumption needing to be made (i.e. the administration cost of intravenous treatment was assumed to be the same regardless of drug delivered). Drug cost parameters were taken from the British National Formulary [30].

The daily cost of inpatient stay (Table 1e) was taken from the NHS Reference Costs 2007-2008 [31], with ventilation and no ventilation being equated with 1 organ supported and 0 organs supported, respectively. The same source was used for the cost of a follow-up appointment with a respiratory consultant [31]. The costs for general ward and respiratory wards were derived from the Personal Social Services Research Unit [32].

\section{Running the model}

The model had a lifetime horizon. Costs did not need to be discounted as no costs were accrued after the first year-it was assumed that there was no possibility of relapse once a patient had been discharged from hospital. Utilities accrued after the first year were discounted at $3.5 \%$ per annum, the recommended rate advocated by the National Institute for Health and Clinical Excellence (NICE) [35].

\section{Cost-utility analysis}

Meropenem and piperacillin/tazobactam were compared for costs and outcomes, i.e. incremental cost per qualityadjusted life year (QALY) gained.
Sensitivity analysis

Parameter uncertainty was assessed in a probabilistic sensitivity analysis (PSA). Here, every variable was given a distribution rather than a fixed probability. Probabilistic sensitivity analysis has become the standard approach recommended by NICE to assess the combined implications of uncertainty in parameters [35]. An alternative method of assessing parameter uncertainty would be to conduct a series of one-way sensitivity analyses, but this approach has been criticised as being relatively insensitive to identify the magnitude of parameter uncertainty [36].

The parameter estimates used in the PSA are presented in Table 3. These were all taken from the same source as the base-case analysis (Table 3a, b, c) with the exception of the drug administration costs (Table $3 \mathrm{~d}$ ) and the daily cost of being on a general/respiratory ward (Table 3e) because the original data had no measure of uncertainty around the mean estimates presented. In these instances, there was an assumed range of values $\pm 50 \%$ of the mean value. There was no need to apply a distribution around the drug acquisition costs, as these are explicit values.

The distributions for the PSA were chosen to reflect the uncertainty in the underlying parameter. For transition probabilities (i.e. Common and efficacy parameters, Table $3 \mathrm{a}, \mathrm{b}$ ), beta distributions were employed to constrain the values estimated for the probabilities between 0 and 1 [36]. Similarly, utility parameters are expected to be constrained between 0 and 1 (death and perfect health), and so beta distributions were employed (Table 3c). The only exception to this was the distribution applied to utilities likely to accrue in critical care as health states considered worse than death are possible-here, a Normal distribution was used.

Relative risks and odds ratios were employed in the model to adjust underlying transition probabilities (Table 3a, b). As these are assumed to follow a Normal distribution on the log scale, a log Normal distribution was applied to be sampled from and the exponential of the result applied to the underlying transition probability.

Like probabilities, cost data are always positive and are based on counts of resource use weighted by unit costs (Table 3d, e). As such, a gamma distribution, which is constrained between 0 and positive infinity, was employed as it can also account for the fact that cost data typically have a skewed distribution [37]. Similarly, where length of stay was employed in the model, for example bed days in HDU, a gamma distribution was used.

Sensitivity analyses were conducted for the primary analysis and the secondary analysis. Furthermore, the effect of changing the age of the patient cohort was investigated using the lower and upper ages from the $95 \%$ confidence interval (Table 2). In addition, the model 
Table 3 Parameter estimates used in the probabilistic sensitivity analysis evaluating the cost-effectiveness of meropenem compared with piperacillin/tazobactam in the treatment of patients with severe pneumonia requiring admission to critical care unit (CCU)

\begin{tabular}{|c|c|c|c|}
\hline \multirow{2}{*}{$\begin{array}{l}\text { Parameter } \\
\text { a Common parameters }\end{array}$} & \multirow[t]{2}{*}{ Distribution } & \multicolumn{2}{|c|}{ Distribution parameters } \\
\hline & & & \\
\hline Patient ventilated & Normal & Mean $=0.53$ & $\mathrm{SD}=0.030000$ \\
\hline Diarrhoea being Clostridium difficile related & Beta & $r=26$ & $\mathrm{n}=373$ \\
\hline Patient cured of $C$. difficile & NA & NA & NA \\
\hline Patient cured of VAP & NA & NA & NA \\
\hline Dying in $\mathrm{CCU}$ if ventilated & Beta & $r=47$ & $n=59$ \\
\hline Dying in CCU if not ventilated & Beta & $r=1$ & $n=97$ \\
\hline Dying in CCU with VAP & Beta & $r=47$ & $n=59$ \\
\hline Adjustment for death from VAP based on age of patient & Log Normal & Mean $=1.678964$ & $\mathrm{SD}=0.605409$ \\
\hline \multirow[t]{4}{*}{ Developing VAP } & Beta & $r=18$ & $n=232$ \\
\hline & Beta & $r=14$ & $n=218$ \\
\hline & Beta & $r=2$ & $n=216$ \\
\hline & Beta & $r=7$ & $n=209$ \\
\hline \multirow[t]{2}{*}{ Adjustment in risk of VAP infection based on prior antibiotic use } & Log Normal & Mean $=-1.237874$ & $\mathrm{SD}=0.446224$ \\
\hline & Log Normal & Mean $=0.382000$ & $\mathrm{SD}=0.195000$ \\
\hline Recovering from VAP & Gamma & $\alpha=1.21$ & $\lambda=0.055000$ \\
\hline Bed days in HDU & Gamma & $\alpha=0.769468$ & $\lambda=0.153894$ \\
\hline Leaving ICU for HDU & Beta & $r=158$ & $n=350$ \\
\hline Leaving ICU for ward & Beta & $r=344$ & $n=164$ \\
\hline Leaving ICU for discharged & Beta & $r=6$ & $n=502$ \\
\hline Leaving HDU for ward & Beta & $r=63$ & $n=2$ \\
\hline Leaving HDU for discharged & Beta & $r=2$ & $n=63$ \\
\hline Discharge from hospital & Gamma & $r=1.1426878$ & $\lambda=0.046076$ \\
\hline \multirow[t]{6}{*}{ Normalised age-related death following discharge from CCU } & Beta & $r=34,324.6$ & $n=2,343,075.4$ \\
\hline & Beta & $r=49,051.7$ & $n=2,017,348.3$ \\
\hline & Beta & $r=71,271.8$ & $n=1,670,828.2$ \\
\hline & Beta & $r=91,848.9$ & $n=1,231,728.2$ \\
\hline & Beta & $r=84,810.4$ & $n=654,689.6$ \\
\hline & Beta & $r=84,467.3$ & $n=297,632.7$ \\
\hline \multirow{9}{*}{$\begin{array}{l}\text { Adjustment for death following discharge from CCU } \\
\text { based on increased risk following CCU admission }\end{array}$} & Log Normal & Mean $=2.725235$ & $\mathrm{SD}=0.142001$ \\
\hline & Log Normal & Mean $=0.936093$ & $\mathrm{SD}=0.210699$ \\
\hline & Log Normal & Mean $=0.717840$ & $\mathrm{SD}=0.221570$ \\
\hline & Log Normal & Mean $=0.518794$ & $\mathrm{SD}=0.232313$ \\
\hline & Log Normal & Mean $=0.412110$ & $\mathrm{SD}=0.236419$ \\
\hline & Log Normal & Mean $=0.198851$ & $\mathrm{SD}=0.260905$ \\
\hline & Log Normal & Mean $=-0.261365$ & $\mathrm{SD}=0.323988$ \\
\hline & Log Normal & Mean $=-0.478036$ & $\mathrm{SD}=0.372096$ \\
\hline & Log Normal & Mean $=0.048790$ & $\mathrm{SD}=0.370579$ \\
\hline Relapsing back into ICU & Beta & $r=7,338.0$ & $n=122,905.0$ \\
\hline Adjustment for death following readmission to ICU & Log Normal & Mean $=2.3978958$ & $\mathrm{SD}=0.158885$ \\
\hline \multicolumn{4}{|l|}{ b Efficacy parameters } \\
\hline Diarrhoea on meropenem & Log Normal & Mean $=0.852600$ & $\mathrm{SD}=0.453000$ \\
\hline Clinical response with meropenem & Log Normal & Mean $=-0.697800$ & $\mathrm{SD}=0.181000$ \\
\hline Diarrhoea on piperacillin/tazobactam & NA & NA & NA \\
\hline Clinical response with piperacillin/tazobactam & NA & NA & NA \\
\hline \multicolumn{4}{|l|}{ c Utility parameters } \\
\hline Utility in critical care centres (ICU and HDU) & Normal & Mean $=-0.402$ & $\mathrm{SD}=0.156$ \\
\hline QALY recovering on ward & Beta & $r=214.2$ & $n=294.9$ \\
\hline
\end{tabular}


Table 3 continued

\begin{tabular}{llll}
\hline Parameter & Distribution & & Distribution parameters \\
\hline QALY 65-74 Males & Beta & $r=0.9$ & $n=1.2$ \\
QALY 65-74 Females & Beta & $r=1.4$ & $n=1.7$ \\
QALY 75 + Males & Beta & $r=1.0$ & $n=1.4$ \\
QALY 75 + Females & Beta & $r=1.3$ & $n=1.8$ \\
d Administration parameters & & & $\lambda=1.542912$ \\
Meropenem & Gamma & $\alpha=16.000000$ & $\lambda=1.542912$ \\
Piperacillin/Tazobactam & Gamma & $\alpha=16.000000$ & $\lambda=2.618658$ \\
Metronidazole & Gamma & $\alpha=16.000000$ & $\lambda=1.805869$ \\
Ceftazidime and gentamicin & Gamma & $\alpha=16.000000$ & \\
e Cost of hospital stay and outpatients appointment parameters & & & $\lambda=0.060233$ \\
Critical care (ICU) & & & $\lambda=0.123278$ \\
$\quad$ Ventilated & Gamma & $\alpha=59.208658$ & $\lambda=0.204255$ \\
$\quad$ Not ventilated & Gamma & $\alpha=141.523692$ & $\lambda=0.133434$ \\
Critical care (HDU) & & & $\lambda=0.070796$ \\
$\quad$ Ventilated & Gamma & $\alpha=144.000000$ & $\lambda=0.854685$ \\
$\quad$ Not ventilated & Gamma & $\alpha=82.328936$ & \\
Respiratory/general ward & Gamma & $\alpha=16.000000$ & \\
Follow-up appointment with Consultant & Gamma & $\alpha=100.852875$ & \\
\hline
\end{tabular}

$N A$ not applicable, $S D$ standard deviation

structure was simplified (i.e. excluding the possibility of VAP, side effects and relapse).

Each PSA was based on 10,000 iterations from a Monte Carlo simulation (where each iteration was assigned a value randomly sampled from the probability distribution around each parameter).

\section{Results}

The results of the deterministic analysis are presented in Table 4. The piperacillin/tazobactam treatment strategy accrues 4.654 QALYs at a cost of $£ 19,978$, while meropenem strategy accrues 4.768 QALYs at a cost of $£ 19,026$. As such, meropenem would be considered a dominant treatment strategy because it accrues more QALYs (0.115) at less cost (-£952) than piperacillin/ tazobactam.

A Markov cohort simulation reveals that, in the meropenem cohort at the end of the model, $26.8 \%$ died in critical care with the remainder dying outside of critical care $(73.2 \%)$. The percentages for piperacillin/tazobactam are $28.6 \%$ and $71.4 \%$, respectively. The additional benefit with meropenem is due to the additional number of patients surviving critical care, with the reduction in cost caused by patients being discharged more quickly from critical care services.

Probabilistic sensitivity analysis (PSA)

The results from the lifetime horizon PSA have similar difference in costs to the base-case model ( $-£ 822$ vs. $-£ 952$, respectively) but a different estimate of benefit (0.015 and 0.115 QALYs, respectively). For the lifetime horizon, the $95 \%$ confidence intervals from the PSA are $£ 13,824-£ 32,018$ (for costs) and 2.153 QALYs to 5.671 QALYs (for effects) for piperacillin/tazobactam and $£ 13,026-£ 31,227$ and 2.157 QALYs to 5.701 QALYs for meropenem.

In order to illustrate the differences in the distributions of costs and effects for the two treatments, the 10,000 iterations from the Monte Carlo simulation have been

Table 4 Costs and quality-adjusted life years (QALYs) associated with meropenem and piperacillin/tazobactam in the treatment of pneumonia in critical care

\begin{tabular}{llllll}
\hline Strategy & Cost & Incremental cost $(\Delta \mathrm{C})$ & Effect & Incremental effect $(\Delta \mathrm{E})$ & ICER $(\Delta \mathrm{C} / \Delta \mathrm{E})$ \\
\hline Piperacillin/tazobactam & $£ 19,978$ & - & 4.654 QALY & - & - \\
Meropenem & $£ 19,026$ & $-£ 952$ & 4.768 QALY & 0.115 QALY & (Dominant) \\
\hline
\end{tabular}


Fig. 2 Cost-effectiveness plane with 10,000 estimates of cost and effect differences between meropenem (MEM) and piperacillin/tazobactam (Pip/Tazo) plotted

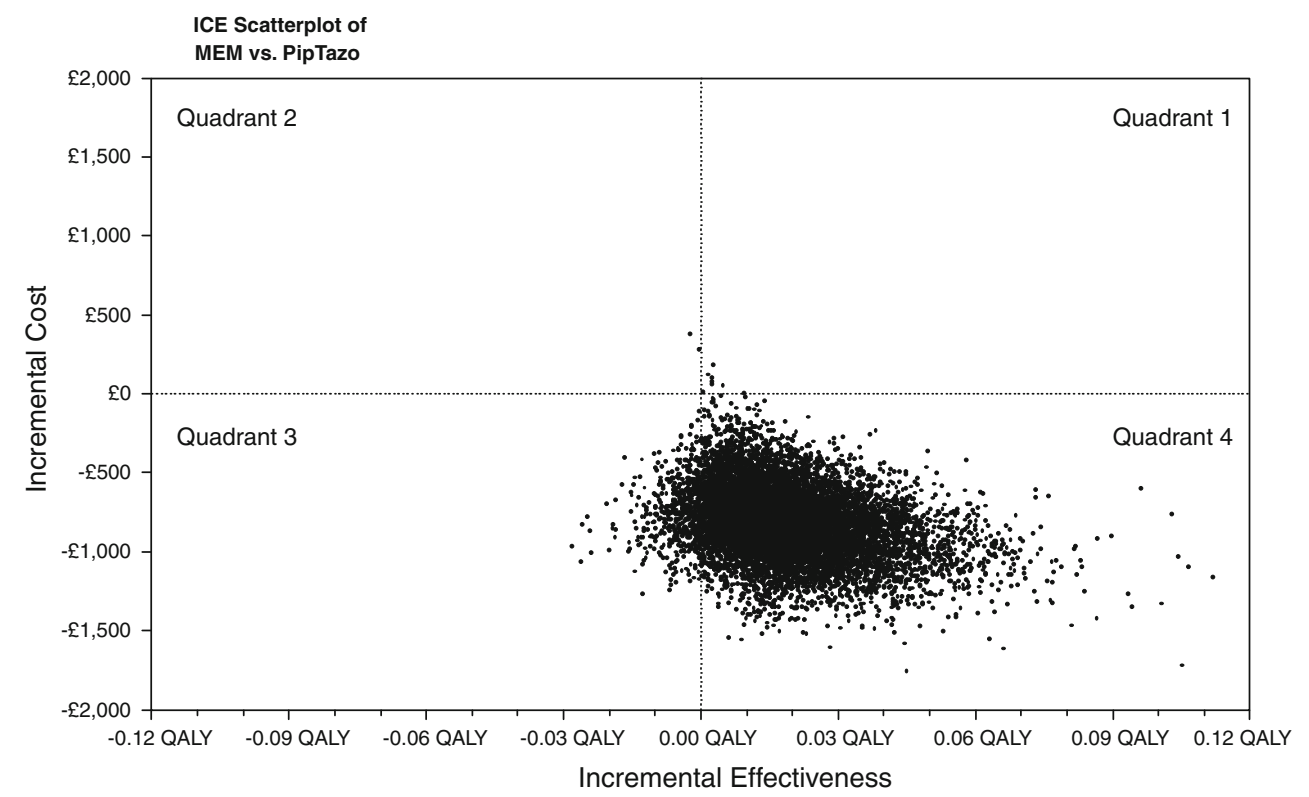

plotted on the cost-effectiveness plane (Fig. 2). The plot demonstrates that $94 \%$ of the iterations lie in quadrant 4 , where meropenem is more effective and less expensive, with $6 \%$ of iterations in quadrant 3 , where meropenem is less effective and less expensive.

The one variable that is not changed in the PSA is the age of the patient entering the model, which is based on the mean value from the patient cohort (Table 2). Two PSAs were conducted using the lower and upper values from the 95\% confidence interval calculated for the age of the patient cohort. Based on the lower age of 66 years, costs and effects become: meropenem-£19,530 (95\% CI: $£ 12,942-£ 31,541)$ and 4.486 QALY (95\% CI: 2.359 QALY to 6.348 QALY); piperacillin/tazobactam$£ 20,334$ (95\% CI: $£ 13,740-£ 32,038$ ) and 4.465 QALY (95\% CI: 2.349 QALY to 6.321 QALY). Of the 10,000 iterations from the Monte Carlo simulation, 94\% lie in quadrant 4 (where meropenem is more effective and less expensive) and $6 \%$ of iterations lie in quadrant 3 (where meropenem is less effective and less expensive). Using the upper age from the $95 \%$ confidence interval of 71 years, costs and effects become: meropenem-£19,309 (£12,929-£30,645) and 3.111 QALY (95\% CI: 1.581 QALY to 4.429 QALY); piperacillin/tazobactam$£ 20,113$ (95\% CI: £13,791-£31,262) and 3.098 QALY (95\% CI: 1.574 QALY to 4.414 QALY). On the costeffectiveness plane, $92 \%$ of iterations lie in quadrant 4 (where meropenem is more effective and less expensive), and $8 \%$ of iterations lie in quadrant 3 (where meropenem is less effective and less expensive).

The simple PSA represents the most basic structure of the Markov model where the risk of patients acquiring VAP, the risk of $C$. difficile-associated diarrhoea and the risk of relapse are not simulated. Here, the costs and effects become: meropenem-£17,747 (95\% CI: $£ 11,079$ $£ 33,071)$ and 5.382 QALY (95\% CI: 2.969 QALY to 7.124 QALY); piperacillin/tazobactam—£18,770 (95\% CI: $£ 12,162-£ 33,729)$ and 5.260 QALY (95\% CI: 2.904 QALY to 6.971 QALY). On the cost-effectiveness plane, $100 \%$ of iterations lie in quadrant 4 , where meropenem is more effective and less expensive.

As meropenem is the dominant treatment strategy, a final PSA was conducted assuming that piperacillin/tazobactam was available at no charge (i.e. an acquisition cost of $£ 0$ rather than a daily cost of $£ 47.37$, Table $18 \mathrm{~d}$ ). Here, costs and effects become: meropenem- $£ 19,398(£ 12,828$ $£ 31,271)$ and 4.028 QALY (95\% CI: 2.145 QALY to 5.680 QALY); piperacillin/tazobactam-£19,877 (95\% CI: $£ 13,306-£ 31,790)$ and 4.009 QALY (95\% CI: 2.138 QALY to 5.650 QALY). On the cost-effectiveness plane, 93\% of iterations lie in quadrant 4 (where meropenem is more effective and less expensive), $6 \%$ lie in quadrant 3 (where meropenem is less effective and less expensive) and $1 \%$ lie in quadrant 1 (where meropenem is more effective and also more expensive).

\section{Discussion}

Our model results show that in the base-case and probabilistic sensitivity analyses, meropenem was more likely to be considered cost-effective than piperacillin/tazobactam. Although the magnitude of benefit and reductions in costs associated with using meropenem rather than piperacillin/ tazobactam differed between analyses, meropenem was considered the dominant treatment. 
The base-case analysis demonstrates a potentially overly positive assessment of likely benefit in term of QALYs compared to most likely value determined by the lifetime PSA (0.115 vs. 0.015 , respectively). This is a good example of how the interplay of parameter uncertainty captured within the average value from the Monte Carlo simulation gives a better estimate of the expected value for the difference in treatments than relying solely on the mean values for parameters. Here, mortality in critical care changes from around $30 \%$ in the base-case analysis to around $40 \%$ in the PSA.

The two PSAs based on age also present predictable results. Both have similar costs to the base-case analysis but quite different estimates of utilities accrued. For younger patients, more QALYs are gained, and for older patients, fewer QALYs are gained resulting in lower and higher estimates of cost per QALYs, respectively, for both treatments assessed. As meropenem is cost-effective in both cases, it could be argued that it will always be costeffective compared to piperacillin/tazobactam regardless of the age of the patient treated.

The accuracy of a model to predict the actual costs and benefits observed in real-life clinical practice is dependent on how closely it reflects that clinical situation. However, from a national policy-making perspective, the evidence required to make a decision to preferentially introduce an intervention compared to an alternative could be simplified into a dichotomous (yes/no) decision where a "national model" would be unlikely to predict the actual costs and benefits observed in any individual locality. The "simple" model is presented as an example of how closely an economic model needs to simulate reality within a decision-making framework. The simple model focuses on the clinical benefit of meropenem and piperacillin/tazobactam, i.e. their ability to cure patients of the pathogen(s) causing pneumonia. However, it does not include the risk of VAP and $C$. difficile-associated diarrhoea identified by the survey of clinicians as important considerations in the treatment of patients with pneumonia in critical care. Nor does it include the risk of relapse.

The results of the simple PSA overestimate the difference in costs and benefits with meropenem compared to piperacillin/tazobactam but do not change the decision that would be made based on the implications of the results. That is, meropenem would be considered the treatment of choice in both the more complicated model and the simple model. Only the impact of the decision to implement a strategy of preferentially using meropenem rather than piperacillin/tazobactam would be affected, i.e. the complex model would more accurately estimate the benefits and costs of implementing a strategy, while the simple model would overestimate the benefits and costs. However, neither would be a completely accurate predictor of the actual costs and benefits realised by a particular critical care unit.
The decision on how closely to simulate the treatment pathway being assessed is difficult. Attempting to assess all outcomes likely to have a major impact on the costs or consequences of treatment is a general guideline. In this context, the more complex model appears to be the correct approach to adopt since a priori the expectation was that the risk of VAP and $C$. difficile-associated diarrhoea could substantially impact on length of stay (costs) and mortality (benefits).

The results of the PSA assessing the impact of piperacillin/tazobactam having a zero acquisition cost provide similar results to the other analyses. There is no change in benefit, and the difference in costs is reduced by $38 \%$ (i.e. from $£ 822$ to $£ 511$ ). However, meropenem remains costsaving compared to piperacillin/tazobactam.

Length of stay in critical care appears to be the main driver in the current example. Remaining in critical care exposes a patient to a high likelihood of dying and of incurring high daily hospitalisation costs. The additional efficacy of meropenem identified in the mixed treatment comparison [11] translates into patients leaving critical care faster than patients receiving piperacillin/tazobactam, with an associated increase in benefit and reduction in costs.

When implementing the findings of this economic evaluation, they should be considered within an understanding of the likely pathogens causing pneumonia in the locality. An assumption within the analysis was that resistance was not an issue and both treatment options could potentially be a cure. If this is not the case, then background resistance patterns should be used as a guide to the most appropriate treatment option.

There are several limitations in our model. Principally, the relative benefit between the two antibiotics is based on a mixed treatment comparison, rather than direct randomised evidence. These differences underpin the key driver in the model of length of stay in critical care, and the associated cost and benefits associated with a reduced length of stay, which result in meropenem being cost-effective compared to piperacillin/tazobactam in the treatment of pneumonia in critical care.

The ideal situation would be for a sufficiently large randomised trial to be conducted to confirm the results of the mixed treatment comparison and so validate the results of the economic evaluation. However, within the context of decision-making, even when considering the value of additional information to reduce parameter uncertainty as advocated by Claxton et al. [36, 38], it would be questionable whether investing in a confirmatory study would be the best use of limited resources. That is, the opportunity cost of the new trial might not be justifiable compared to funding research into an area where there is greater uncertainty. In addition, there is the impact of delaying 
actions based on our findings of clinical benefit for patients and cost savings for the NHS (by preferentially using meropenem rather than piperacillin/tazobactam where appropriate).

A further limitation of our study is the lack of HRQL in this area. No data were available from prospective research. However, as there is no evidence to suggest that patients experience a different level of HRQL while being treated with either antibiotic, or that patients experience a different level of HRQL after completing treatment dependent on which antibiotic they received, it would be unlikely that more accurate data would change the finding that meropenem accrues more QALYs than piperacillin/ tazobactam. While more accurate utility values would affect the estimates produced by the evaluation, they would not affect the general conclusion that favours meropenem.

Similarly, other assumptions made for the parameter estimates used in the model, such as the uncertainty around the cost of administration and the daily cost of being on a general/respiratory ward, are unlikely to have such an impact on the results of the model to change the implication of the results.

Another limitation is the use of HES data for the estimates of patients leaving locations of care (i.e. ICU, HDU and general/respiratory ward). However, a recent audit conducted by the Audit Commission found that HES data were appropriately coded in approximately $90 \%$ of cases [39]. In addition, the variability around the estimates used in the PSA (Table 3) appears to contain a range of values that would appear to capture all plausible options, for example the extreme range of values for patients leaving ICU include the majority of patients going to HDU and all patients going from ICU to the general/respiratory ward. The HES data also matched well the expectations of the clinical experts who were surveyed and so in this sense had strong "face validity".

Finally, the data for each parameter included in the evaluation could have been individually subject to a systematic review of the literature to ensure that the best available evidence was used. In this instance, we took a pragmatic decision based on the expected incremental benefit of additional research balanced with the investment in time required to perform it. This is no different from the judgments involved in determining how exhaustive a typical systematic review of efficacy would be. As with any systematic review, it is important that the reporting of the research is clear and detailed, allowing readers to decide how thorough they believe the review to have been.

\section{Conclusions}

The results of this cost-effectiveness analysis evaluation suggest that the substantial benefits associated with meropenem over piperacillin/tazobactam in the treatment of pneumonia in critical care also translate into cost savings for UK critical care services, making it a dominant treatment strategy. This result was robust to plausible changes in model parameters assessed in a PSA.

Acknowledgments When this research was conducted SJE was an employee of AstraZeneca UK Ltd, the distributor of Meronem ${ }^{\circledR}$, who provided some support for his work on this economic evaluation. SW and MJC are both funded by the UK Department of Health and received no support or funding from AstraZeneca UK Ltd.

\section{References}

1. Welch, C.: Personal Communication (February 2009). Case Mix Programme Database (ICNARC, Tavistock House, Tavistock Place, London WC1H 9HR)

2. Armitage, K., Woodhead, M.: New guidelines for the management of adult community-acquired pneumonia. Curr. Opin. Infect. Dis. 20, 170-176 (2007)

3. American Thoracic Society.: Hospital-acquired pneumonia in adults: diagnosis, assessment of severity, initial antimicrobial therapy, and preventative strategies. Am. J. Respir. Crit. Care. Med. 153, 1711-1725 (1995)

4. American Thoracic Society.: Guidelines for the management of adults with community-acquired pneumonia. Am. J. Respir. Crit. Care. Med. 163 1730-1750 (2001)

5. American Thoracic Society.: Guidelines for the management of adults with hospital-acquired, ventilator-associated, and healthcare associated pneumonia. Am. J. Respir Crit. Care Med. 171 388-416 (2005)

6. British Thoracic Society.: BTS guideline for the management of community-acquired pneumonia in adults-2004 update. Available at: www.brit-thoracic.org.uk/Clinical-Information/ Pneumonia/Pneumonia-Guidelines.aspx (last Accessed Nov 2009)

7. Bodmann, K.F.: Current guidelines for the treatment of severe pneumonia and sepsis. Chemotherapy 51, 227-233 (2005)

8. Edwards, S.J., Campbell, H.E., Plumb, J.M.: A cost-utility analysis comparing meropenem with imipenem plus cilastatin in the treatment of severe infections in intensive care. Eur. J. Health Econ. 7, 72-78 (2006)

9. Edwards, S.J., Clarke, M.J., Wordsworth, S., et al.: Carbapenems versus other beta-lactams in treating severe infections in intensive care: a systematic review of randomised controlled trials. Eur. J. Clin. Microbiol. Infect. Dis. 27, 531-543 (2008)

10. Edwards, S.J., Clarke, M.J., Wordsworth, S., et al.: Indirect comparisons of treatments based on systematic reviews of randomised controlled trials. Intern. J. Clin. Pract. 63, 841-854 (2009)

11. Edwards, S.J., Clarke, M.J., Wordsworth, S., et al.: Carbapenems versus other beta-lactams in the treatment of hospitalised patients with infection: a mixed treatment comparison. Curr. Med. Res. Opin. 25, 251-261 (2009)

12. Weber, R.J., Kane, S.L., Oriolo, V.A., et al.: Impact of intensive care unit (ICU) drug use on hospital costs: a descriptive analysis, with recommendations for optimizing ICU pharmacotherapy. Crit. Care Med. 31(1 Suppl), S17-S24 (2003)

13. Rello, J., Bodi, M., Mariscal, D., et al.: Microbiological testing and outcome of patients with severe community-acquired pneumonia. Chest 123, 174-180 (2003)

14. McFarland, L.V., Surawicz, C.M.: Risk factors for Clostridium difficile carriage, $C$ difficile-associated diarrhea in a cohort of hospitalised patients. J. Infect. Dis. 162, 678-684 (1990) 
15. Poutanen, S.M., Simor, A.E.: Clostridium difficile-associated diarrhea in adults. CMAJ 171, 51-58 (2004)

16. Chastre, J., Wolff, M., Fagon, J.-Y., et al.: Comparison of 8 vs 15 days of antibiotic therapy for ventilator-associated pneumonia in adults. JAMA 290, 2588-2598 (2003)

17. Rello, J., Ollendorf, D.A., Oster, G., et al.: Epidemiology and outcomes of ventilator-associated pneumonia in a large US database. Chest 122, 2115-2121 (2002)

18. Hyllienmark, P., Gårdlund, B., Persson, J.-O., et al.: Nosocomial pneumonia in the ICU: a prospective cohort study. Scand. J. Infect. Dis. 39, 676-682 (2007)

19. Rello, J., Diaz, E., Roque, M., et al.: Risk factors for developing pneumonia within 48 hours of intubation. Am. J. Respir. Crit. Care Med. 159, 1742-1746 (1999)

20. Das, R.: Critical care admissions for pneumonia in England. Value Health 7, A431 (2009)

21. Office for National Statistics.: Mortality statistics-death registered in 2007. Newport: ONS, 2007. Available at: www. statistics.gov.uk/statbase/Product.asp?vlnk=15096 (last Accessed July 2009)

22. Wright, J.C., Plenderleith, L., Ridley, S.A.: Long-term survival following intensive care: subgroup analysis and comparison with the general population. Anaesthesia 58, 637-642 (2003)

23. Rosenberg, A.L., Watts, C.: Patients readmitted to ICUs: a systematic review of risk factors and outcomes. Chest 118, 492-502 (2000)

24. Rosenberg, A.L., Hofer, T.P., Hayward, R.A., et al.: Who bounces back? Physiological and other predictors of intensive care unit readmission. Crit. Care Med. 29, 511-518 (2001)

25. Schmitt, D.V., Leitner, E., Welte, T., et al.: Piperacillin/tazobactam vs imipenem/cilastatin in the treatment of nosocomial pneumonia-a double blind prospective multicentre study. Infection 34, 127-134 (2006)

26. Kind, P., Hardman, G., Macran, S.: UK Population norms for EQ5D. Discussion Paper 172. University of York, Centre for Health Economics (1999)

27. Eddleston, J.M., White, P., Guthrie, E.: Survival, morbidity, and quality of life after discharge from intensive care. Crit. Care Med. 28, 2293-2299 (2000)

28. Ara, R., Brazier, J.: Deriving an algorithm to convert the eight mean SF-36 dimension scores into a mean EQ-5D preference- based score from published studies (where patient level data are not available). Value in Health 11, 1131-1143 (2008)

29. Van Zanten, A.R.H., Engrlfriet, P.M., Van Dillen, K., et al.: Importance of nondrug costs of intravenous antibiotic therapy. Crit. Care 7, 184-190 (2003)

30. British National Formulary No. 56 (September 2008). Available at: www.bnf.org/bnf/bnf/current/index.htm (last accessed December 2008)

31. National Health Service.: Reference Costs 2007/8. London: Department of Health, Feb 2008. Available at: www.dh.gov.uk/ en/Publicationsandstatistics/Publications/PublicationsPolicyAnd Guidance/DH_098945 (last Accessed July 2009)

32. Curtis L.: Unit Costs of Health and Social Care 2008. PSSRU, University of Kent at Canterbury (2008)

33. Sun, X., Faunce, T.: Decision-analytical modelling in health-care economic evaluations. Eur. J. Health Econ. 9, 313-323 (2008)

34. American Thoracic Society.: Understanding Costs and CostEffectiveness in Critical Care Report from the Second American Thoracic Society Workshop on Outcomes Research. Am. J. Respir Crit. Care Med. 165, 540-550 (2002)

35. National Institute for Health and Clinical Excellence.: Guide to the method of technology appraisal. National Institute for Health and Clinical Excellence, June 2008. Available at:www.nice.org. uk/media/B52/A7/TAMethodsGuideUpdatedJune2008.pdf (last Accessed March 2010)

36. Claxton, K., Sculpher, M., McCabe, C., et al.: Probabilistic sensitivity analysis for NICE technology assessment: not an optional extra. Health Econ. 14, 339-347 (2005)

37. Briggs, A.H., Gray, A.M.: The distribution of health care costs and their statistical analysis for economic evaluation. J. Health Serv. Res. Policy 3, 233-245 (1998)

38. Claxton, K.: The irrelevance of inference: a decision-making approach to the stochastic evaluation of health technologies. J. Health Econ. 18, 341-364 (1999)

39. Audit Commission.: PbR Data Assurance Framework 2007/08findings from the first year of the national clinical coding audit programme. Health National Report, August 2008. Audit Commission, London (2008) 